\title{
A New Micro Method for the Hemosedimentation with 0.05 c.c. of Blood.
}

\author{
3rd Hematological Paper.
}

By

\section{Matuiti Yoshida.}

(吉田 松一)

(From the Department of Pediatries, Faculty of Medicine, Tohoku Imperial University, Sendai.

Director: Prof. A. Sato.)

\section{Introduction.}

Blood sedimentation test is not so widely used, within my knowledge, in pediatrics as in adult medicine. The reason is, I believe, very simple: the amount of blood necessary for the test is too large $\mathrm{e}^{1)}$ in the case of children. Indeed, there are such micro-methods, as Linzenmeier's' and Langer-Schmidt's ${ }^{3)}$ ones. $^{5}$ But I had been trying a micro method with a very small amount of blood. In the present paper I desire to describe my micro hemosedimeter which requires only 0.05 c.c. of blood.

\section{My Own Hemosedimeter.}

1. The amount of blood required is only 0.05 c.c. or $\frac{1}{20}$ c.c.

2. Micro pipette. The pipette is about $9.5 \mathrm{~cm}$ long and has an internal diameter $1.3 \mathrm{~m} . \mathrm{m}$. It has on it three marks : $\mathrm{B}, \mathrm{O}$ and $\mathrm{C}$.

1) Ci. Westergren's hemosedimeter: A. Westergren, Ergeb. d. inn. Med., $1924,26,577$.

2) Linzenmeier Münch. med. Wschr., 1925, 5.

3) H. L a nger and W. S chmidt, Zschr. f. Kinderh., 1926, 41, 72.

$\S$ There is also K.S a to's micro-method, which requires 0.2 c.o. of blood i.e. a very large amount of blood in contrast to 0.05 c.c. of blood required in my own method. Cf K. S a t o, Tokyo Iji Shinshi, 1931, No. 2750, 2517; Iji Koron, 1932, No. 1066, 11. 
Fig. 1. Diagram of $\mathrm{Y}_{0-}$ shida's micro pipette.

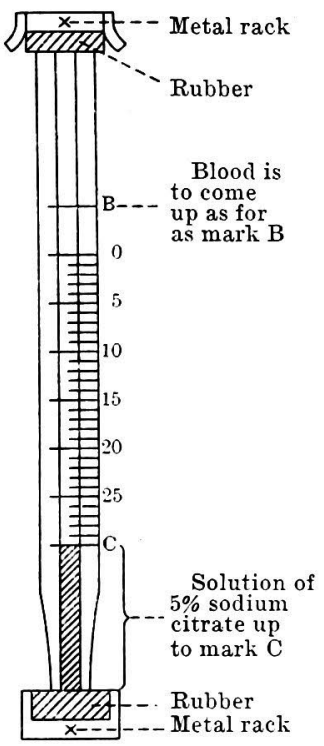

Fig. 2. Blood taking.

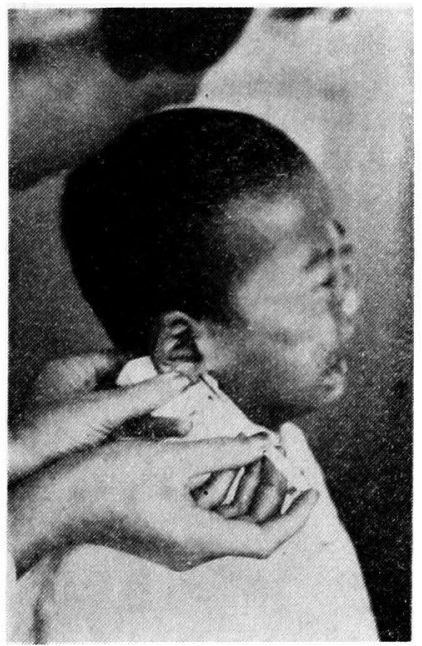

The pipette between $\mathrm{O}$ and $\mathrm{C}$ is divided into 30 equal parts, each part being 1 m.m. in length (Cf. Fig. I). The mark B is a little above the mark $O$. Fig. I will show the other details of the apparatus.

3. Solution required. $5 \%$ aquous solution of sodium citrate. 5 grms. of sodium citrate neutral; $\mathrm{C}_{3} \mathrm{H}_{4} \mathrm{OH}-$ (COO.Na) $)_{3} 5 \frac{1}{2} \mathrm{H}_{2} \mathrm{O}$ neutral is dissolved in 100 c.c. of distilled water.

4. A white celloid disc with a hollow place in the central part for mixing blood (Cf. Fig. III).

5. The whole apparatus is contained in a metal rack (Cf. Fig. II). which may be kept in a pocket and conveyed anywhere (Cf. Fig. IV).

\section{Technique.}

The pipette should of course be epiously cleaned. It is by far better (especially for a beginner) to fill the citrate up to the mark $\mathrm{B}$, and only then to let it fall down to the mark $\mathrm{C}$ of the pipette by means of capillarity and then make an incision by use of a knife or Frank's needle on an ear-lobe* or on a finger tip. If a blood drop comes out, bring the point of the pipette into contact with the drop, first holding it almost vertical and then gradually bring it to a horizontal position, keeping the point in contact with the blood drop. During the process, blood will come into the pipette by means of capillarity (Cf. Fig. II). As soon as the blood column reaches the mark $B$, take the pipette away from the

* It is still better to apply warm water to the ear for a few minutes. 
Fig. 3. Blood mixing.

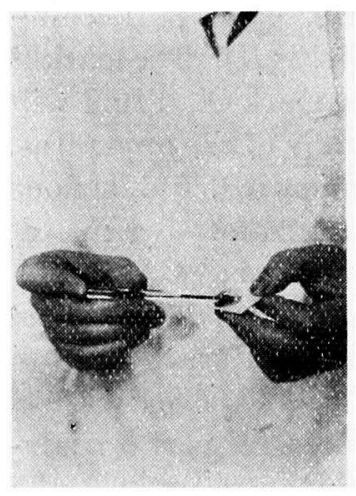

blood drop and wipe any blood off the point.

Then blow the whole contents of the pipette into the hollow place of the celloid disc and mix well with the point of the pipette. Then fill the pipette again with the mixture up to the mark $O$ (not up to the mark B) by capillarity, the technique of filling it being the same as above described (Cf. Fig. III).

Then bring the pipette into the rack and keep it between the two pieces out of gummi (Cf. Figs. I and IV).

The column of clear serum above the setting red blood cells is read in millimeters at the termination of one hour; if necessary in clinical observation, a reading at 15,30 , 45 and 60 minutes after blood taking ought to be read.

\section{Result of Experiment.}

1. Comparison between Langer-Schmidt's and my own methods (Cf. Tab. I).

Here I want to compare the results obtained by the two micro methods, Langer-Schmidt's and the author's. It will be seen from Table I the averaged differences show only $\pm 0.4 \mathrm{~mm}$. in 15 minutes, $\pm 0.6 \mathrm{~mm}$. in 30 minutes, $\pm 0.7 \mathrm{~mm}$. in 45 minutes and $\pm 0.7 \mathrm{~mm}$. in 60 minutes. The result is, I believe, sufficient to show that my own method can be used for measuring the sedimentation value instead of LangerSchmidt's method, especially in pediatric practice; because my own method requires only 0.05 c.c. of blood in contrast to 0.12 c.c. of blood required in Langer-Schmidt's method. Besides in my method the apparatus complete
Fig. 4. How to set pipettes in the metal rack.

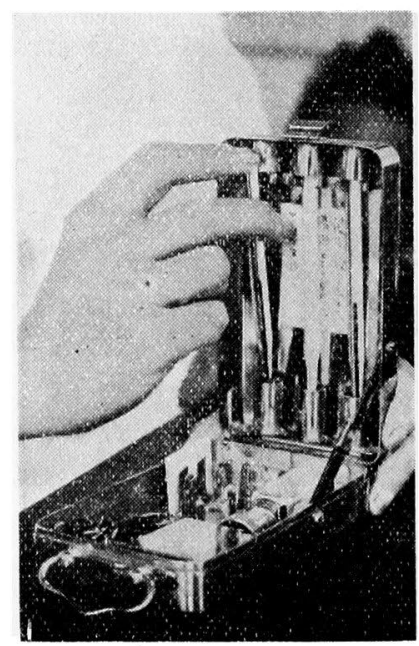


Fig. 5. Comparison between author's method and Westergren's.
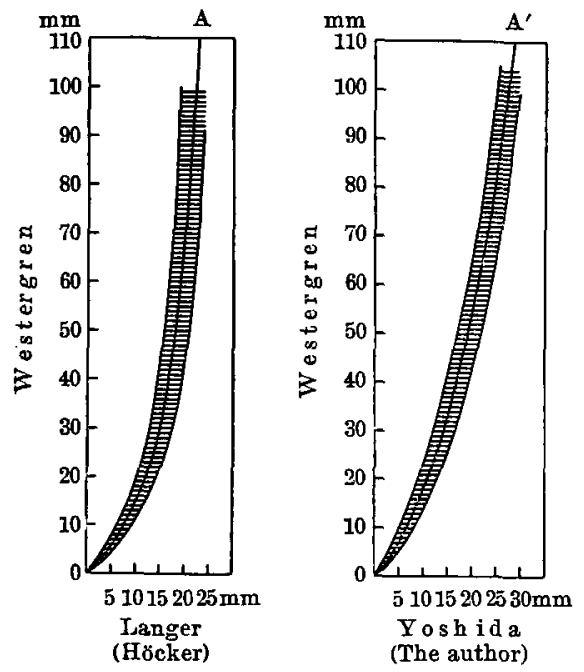

A , Javeraged values may be carried anywhere in the pocket.** Furthermore, it is rather a nuisance with a micro method of blood sedimentation to have blood easily clotted. It is for this reason that Schierlitz ${ }^{4}$ wrote in detail about the avoidance of such a technical failure. In my own method there is no fear of blood clotting, because one can obtain the small amount of 0.05 c.c. blood very quickly even in the case of a small baby.

2. Comparison between Westergren's and my own method (Cf. Tab. II and Fig. V).

Höcker, ${ }^{5)}$ A chard, Lévy and Guthman $n^{6)}$ made a comparison between the results of Langer-Schmidt's method and those of Westergren's. Höcker made a diagram as figured in Diagram (Cf. Fig. V), in which the result of my own comparison between Westergren's and my method will be shown. The sedimentation test was performed at a room temperature of $28^{\circ} \mathrm{C}$. (Cf. Diagram I). From the latter, the corresponding readings of Westergren's and my own method will be found.

3. Appreciation of the readings in my own method (Cf. Tables III and IV).

Here a preliminary trial is made of the clinical appreciation of the readings in my micro method. Clinical cases may be classified into 5 classes according to the blood sedimentation rate namely:-

1. Subnormal (=protracted)............under $2 \mathrm{~mm}$. in 1 hour.

2. normal ..............................4 $4 \mathrm{~mm}$. in 1 hour. (Sometimes 9-11 mm. in 1 hour in healthy cases).

3. Slightly accelerated $12-15 \mathrm{~mm}$. in 1 hour.

4. Moderately accelerated .............15-20 mm. in 1 hour.

5. Strongly accelerated 21-25-30 $\mathrm{mm}$. in 1 hour.

** The pipette in my method is not placed in a tube of mercury as in LangerSchmidt's method.

4) K. Schierlitz, Münch. med. Wschr., 1930, 356.

5) H. Hö c k er, Münch. med. Wschr., 1931, 1556.

6) Achard, Lévg and Guthmann, Le Sang, 1933, 6, 557. 


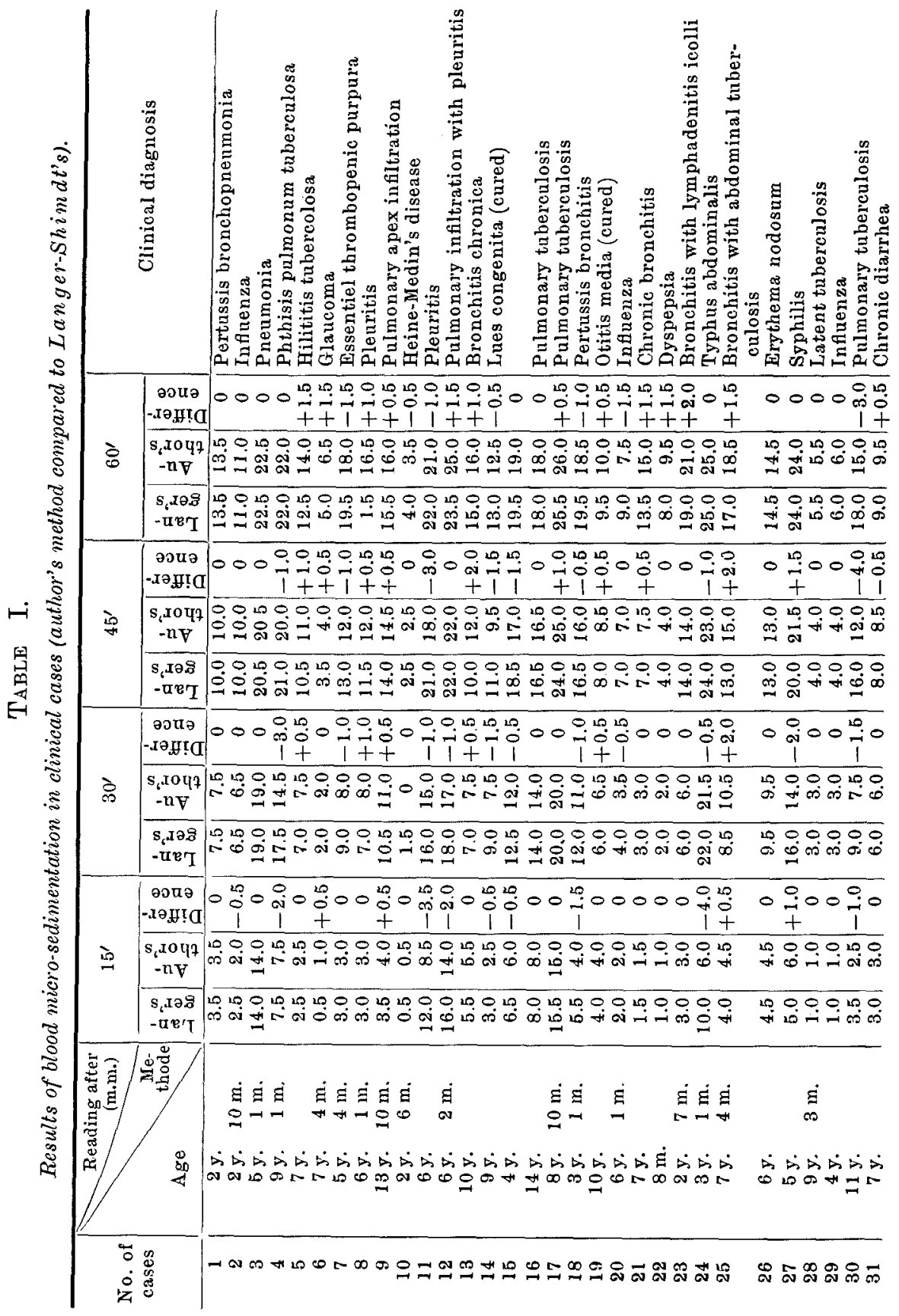




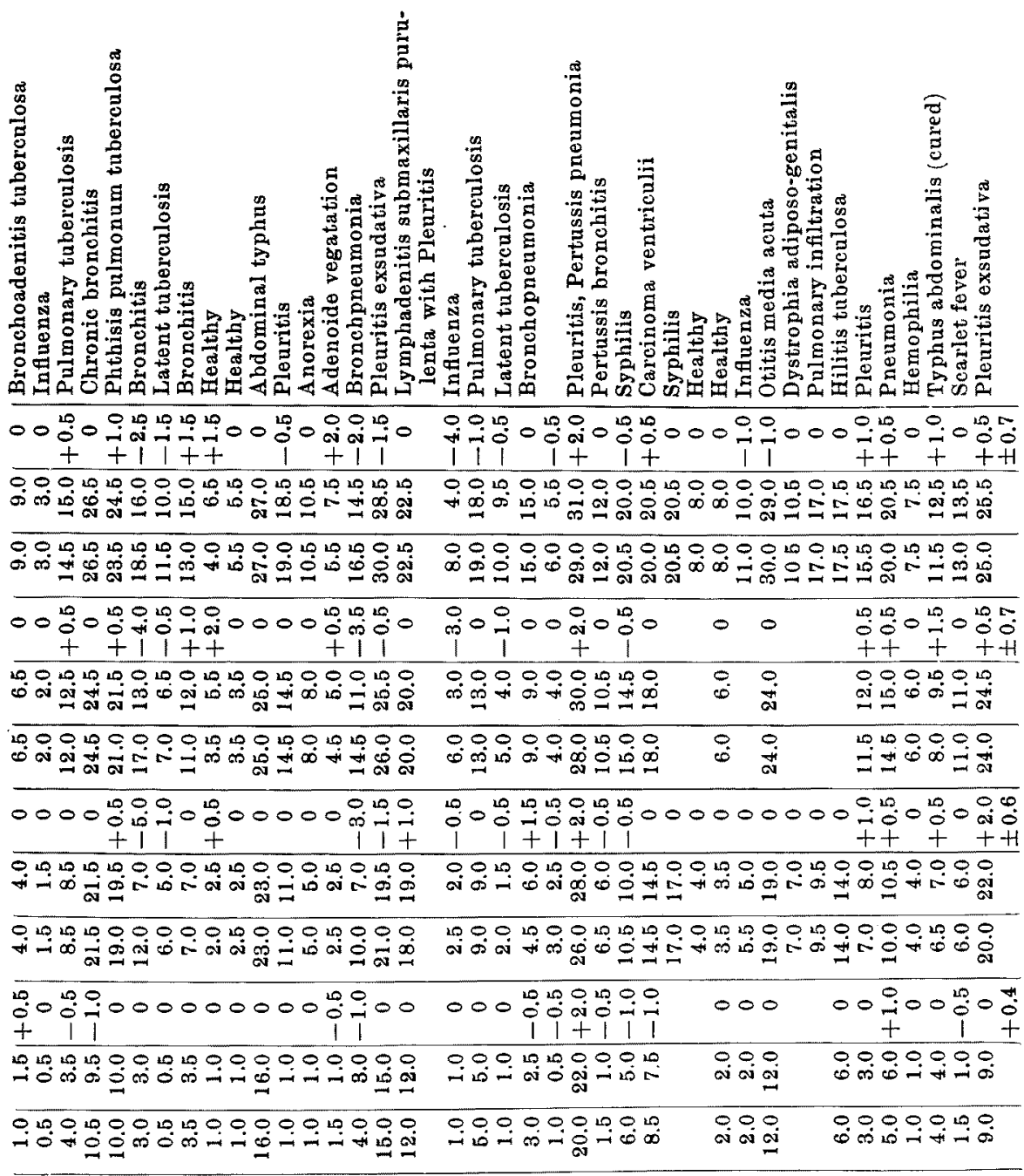

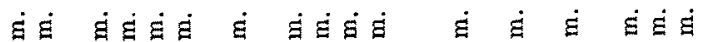

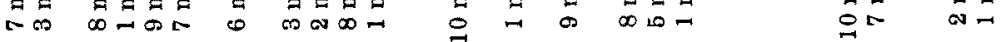

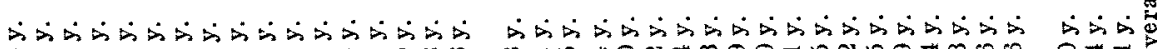

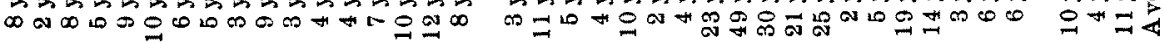

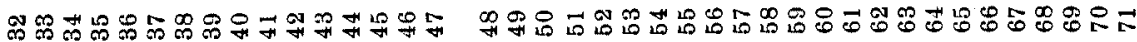


TABLE II.

Comparison of the results of author's method and We stergren's method.

\begin{tabular}{|c|c|c|c|c|c|c|c|c|c|}
\hline \multirow[b]{2}{*}{ 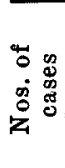 } & \multirow[b]{2}{*}{ Time } & \multicolumn{2}{|c|}{$\begin{array}{c}\text { Blood sedimentation, } \\
\text { m.m. }\end{array}$} & \multirow[b]{2}{*}{$\begin{array}{l}\text { Age. Sex. } \\
\text { Diagnosis }\end{array}$} & \multirow[b]{2}{*}{ 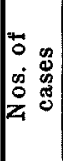 } & \multirow[b]{2}{*}{ Time } & \multicolumn{2}{|c|}{$\begin{array}{c}\text { Blood sedimentation, } \\
\mathrm{m} . \mathrm{m} .\end{array}$} & \multirow[b]{2}{*}{$\begin{array}{l}\text { Age. Sex. } \\
\text { Diagnosis }\end{array}$} \\
\hline & & $\begin{array}{l}\text { Authro's } \\
\text { micro- } \\
\text { method }\end{array}$ & $\begin{array}{l}\text { Wester- } \\
\text { gren's } \\
\text { Macro- } \\
\text { method }\end{array}$ & & & & $\begin{array}{l}\text { Authro's } \\
\text { micro- } \\
\text { method }\end{array}$ & $\begin{array}{l}\text { Wester- } \\
\text { gren's } \\
\text { Macro- } \\
\text { method }\end{array}$ & \\
\hline 1 & $\begin{array}{l}15^{\prime} \\
30^{\prime} \\
45^{\prime} \\
60^{\prime} \\
\mathbf{7 5 ^ { \prime }} \\
90^{\prime}\end{array}$ & $\begin{array}{r}7.5 \\
15.0 \\
18.0 \\
19.0 \\
20.0 \\
21.5\end{array}$ & $\begin{array}{l}15.0 \\
28.0 \\
35.0 \\
53.0 \\
67.0 \\
72.0\end{array}$ & $\begin{array}{c}14 \mathrm{y} .5 \mathrm{~m} . \\
\text { Pulmonary } \\
\text { tuber- } \\
\text { culosis }\end{array}$ & 9 & $\begin{array}{r}15^{\prime} \\
30^{\prime} \\
45^{\prime} \\
60^{\prime} \\
90^{\prime} \\
120^{\prime}\end{array}$ & $\begin{array}{r}3.0 \\
9.0 \\
14.0 \\
19.0 \\
22.0 \\
24.0\end{array}$ & $\begin{array}{r}4.5 \\
13.0 \\
24.0 \\
37.0 \\
55.0 \\
68.0\end{array}$ & $\begin{array}{c}7 \mathrm{y} .4 \mathrm{~m} \\
\text { Bronchitis }\end{array}$ \\
\hline & $\begin{array}{l}105^{\prime} \\
120^{\prime}\end{array}$ & $\begin{array}{l}22.5 \\
23.5\end{array}$ & $\begin{array}{l}76.0 \\
79.0\end{array}$ & & & $\begin{array}{l}15^{\prime} \\
30^{\prime}\end{array}$ & $\begin{array}{l}2.5 \\
7.5\end{array}$ & $\begin{array}{r}6.0 \\
14.0\end{array}$ & \\
\hline 2 & $\begin{array}{l}15^{\prime} \\
30^{\prime} \\
45^{\prime} \\
60^{\prime}\end{array}$ & $\begin{array}{l}2.0 \\
3.0 \\
5.0 \\
7.5\end{array}$ & $\begin{array}{r}2.0 \\
7.0 \\
12.0 \\
18.0\end{array}$ & $\begin{array}{l}23 \mathrm{y} . \\
\text { Healthy }\end{array}$ & 10 & $\begin{array}{r}45^{\prime} \\
60^{\prime} \\
90^{\prime} \\
120^{\prime}\end{array}$ & $\begin{array}{l}12.0 \\
15.0 \\
17.5 \\
18.5\end{array}$ & $\begin{array}{l}26.0 \\
41.0 \\
53.0 \\
59.0\end{array}$ & $\begin{array}{l}11 \mathrm{~g} . \\
\text { Pleuritis }\end{array}$ \\
\hline & $\begin{array}{r}90^{\prime} \\
120^{\prime}\end{array}$ & $\begin{array}{l}10.0 \\
13.0\end{array}$ & $\begin{array}{l}\mathbf{2 3 . 0} \\
\mathbf{2 8 . 0}\end{array}$ & & & $\begin{array}{l}15^{\prime} \\
30^{\prime}\end{array}$ & $\begin{array}{l}3.0 \\
7.0\end{array}$ & $\begin{array}{r}4.0 \\
13.0\end{array}$ & \\
\hline 3 & $\begin{array}{r}15^{\prime} \\
30^{\prime} \\
45^{\prime} \\
60^{\prime} \\
90^{\prime} \\
120^{\prime}\end{array}$ & $\begin{array}{r}2.0 \\
3.0 \\
7.0 \\
11.0 \\
14.0 \\
17.0\end{array}$ & $\begin{array}{r}1.5 \\
7.0 \\
12.0 \\
20.0 \\
21.0 \\
38.0\end{array}$ & $\begin{array}{l}28 \mathrm{y} . \\
\text { Healthy }\end{array}$ & 11 & $\begin{array}{r}45^{\prime} \\
60^{\prime} \\
75^{\prime} \\
90^{\prime} \\
105^{\prime} \\
120^{\prime}\end{array}$ & $\begin{array}{l}13.0 \\
16.0 \\
19.5 \\
20.0 \\
22.0 \\
23.0\end{array}$ & $\begin{array}{l}27.0 \\
38.0 \\
48.0 \\
53.0 \\
60.0 \\
63.0\end{array}$ & $\begin{array}{c}12 \mathrm{y} . \\
\text { Pulmonary } \\
\text { tuber- } \\
\text { culosis }\end{array}$ \\
\hline 4 & $\begin{array}{r}15^{\prime} \\
30^{\prime} \\
45^{\prime} \\
60^{\prime} \\
90^{\prime} \\
120^{\prime}\end{array}$ & $\begin{array}{r}2.0 \\
6.5 \\
11.5 \\
15.5 \\
18.0 \\
21.5\end{array}$ & $\begin{array}{r}4.0 \\
12.0 \\
20.0 \\
40.0 \\
52.0 \\
68.0\end{array}$ & $\begin{array}{c}30 \mathrm{y} \\
\text { Menstrua- } \\
\text { tion }\end{array}$ & 12 & $\begin{array}{r}15^{\prime} \\
30^{\prime} \\
45^{\prime} \\
60^{\prime} \\
90^{\prime} \\
120^{\prime}\end{array}$ & $\begin{array}{r}1.5 \\
3.0 \\
7.0 \\
10.5 \\
13.5 \\
17.0\end{array}$ & $\begin{array}{r}1.5 \\
4.0 \\
11.0 \\
21.0 \\
29.0 \\
51.0\end{array}$ & $\begin{array}{c}7 \text { y. } \\
\text { Chronic } \\
\text { bronchitis }\end{array}$ \\
\hline 5 & $\begin{array}{r}15^{\prime} \\
30^{\prime} \\
45^{\prime} \\
60^{\prime} \\
90^{\prime} \\
120^{\prime}\end{array}$ & $\begin{array}{r}7.5 \\
14.5 \\
18.0 \\
20.5 \\
23.0 \\
25.0\end{array}$ & $\begin{array}{r}6.5 \\
21.0 \\
32.0 \\
47.0 \\
63.0 \\
75.0\end{array}$ & $\begin{array}{c}49 \mathrm{y} \\
\text { Carcinoma } \\
\text { ventriculi }\end{array}$ & 13 & $\begin{array}{r}15^{\prime} \\
30^{\prime} \\
45^{\prime} \\
60^{\prime} \\
90^{\prime} \\
120^{\prime}\end{array}$ & $\begin{array}{r}2.0 \\
5.0 \\
7.5 \\
11.0 \\
15.5 \\
17.0\end{array}$ & $\begin{array}{r}2.0 \\
5.5 \\
12.0 \\
17.5 \\
36.0 \\
46.0\end{array}$ & $\begin{array}{c}12 \mathrm{y} . \\
\text { Pulmonary } \\
\text { tuber- } \\
\text { culosis }\end{array}$ \\
\hline 6 & $\begin{array}{r}15^{\prime} \\
30^{\prime} \\
45^{\prime} \\
60^{\prime} \\
90^{\prime} \\
120^{\prime}\end{array}$ & $\begin{array}{r}3.0 \\
8.0 \\
12.0 \\
15.0 \\
19.0 \\
20.0\end{array}$ & $\begin{array}{r}2.5 \\
10.5 \\
19.0 \\
29.0 \\
46.0 \\
61.0\end{array}$ & $\begin{array}{l}3 \text { y. } 1 \mathrm{~m} . \\
\text { Bronch- } \\
\text { pneu- } \\
\text { monia }\end{array}$ & 14 & $\begin{array}{r}15^{\prime} \\
30^{\prime} \\
45^{\prime} \\
60^{\prime} \\
90^{\prime} \\
120^{\prime}\end{array}$ & $\begin{array}{r}2.0 \\
4.0 \\
7.0 \\
9.0 \\
13.0 \\
17.0\end{array}$ & $\begin{array}{r}2.0 \\
3.0 \\
6.5 \\
8.5 \\
13.0 \\
27.0\end{array}$ & $\begin{array}{l}6 \mathrm{y} .2 \mathrm{~m} . \\
\text { Latent } \\
\text { tuber- } \\
\text { culosis }\end{array}$ \\
\hline 7 & $\begin{array}{r}15^{\prime} \\
30^{\prime} \\
45^{\prime} \\
60^{\prime} \\
90^{\prime} \\
120^{\prime}\end{array}$ & $\begin{array}{r}0.6 \\
3.0 \\
5.5 \\
9.0 \\
14.0 \\
18.0\end{array}$ & $\begin{array}{r}2.0 \\
5.0 \\
8.5 \\
15.0 \\
32.0 \\
45.0\end{array}$ & $\begin{array}{c}23 \mathrm{y} . \\
\text { Influenza }\end{array}$ & 15 & $\begin{array}{r}15^{\prime} \\
30^{\prime} \\
45^{\prime} \\
60^{\prime} \\
75^{\prime} \\
90^{\prime} \\
120^{\prime}\end{array}$ & $\begin{array}{r}2.5 \\
6.0 \\
11.5 \\
13.5 \\
14.5 \\
14.5 \\
18.0\end{array}$ & $\begin{array}{r}4.0 \\
12.0 \\
26.0 \\
32.0 \\
40.0 \\
40.0 \\
56.0\end{array}$ & $\begin{array}{c}9 \mathrm{y} . \\
\text { Pulmonary } \\
\text { tuber- } \\
\text { culosis }\end{array}$ \\
\hline 8 & $\begin{array}{r}15^{\prime} \\
30^{\prime} \\
45^{\prime} \\
60^{\prime} \\
90^{\prime} \\
120^{\prime}\end{array}$ & $\begin{array}{r}2.0 \\
7.5 \\
11.0 \\
14.0 \\
18.0 \\
21.0\end{array}$ & $\begin{array}{r}2.5 \\
10.0 \\
17.0 \\
21.0 \\
35.0 \\
43.0\end{array}$ & $\begin{array}{c}10 \mathrm{y} . \\
\text { Diphtheric } \\
\text { angina }\end{array}$ & 16 & $\begin{array}{r}15^{\prime} \\
30^{\prime} \\
45^{\prime} \\
60^{\prime} \\
90^{\prime} \\
120^{\prime}\end{array}$ & $\begin{array}{r}1.5 \\
4.0 \\
10.0 \\
16.0 \\
19.0 \\
20.0\end{array}$ & $\begin{array}{r}3.0 \\
6.5 \\
22.0 \\
50.0 \\
72.0 \\
87.0\end{array}$ & $\begin{array}{c}12 \mathrm{y} .1 \mathrm{~m} . \\
\text { Pleuro- } \\
\text { peritonitis }\end{array}$ \\
\hline
\end{tabular}


TABLE III.

Blood micro-sedimentation in different cases.

\begin{tabular}{|c|c|c|c|c|c|c|}
\hline \multirow{2}{*}{$\begin{array}{c}\text { Nos. } \\
\text { of } \\
\text { cases }\end{array}$} & \multirow{2}{*}{ Age } & \multicolumn{4}{|c|}{$\begin{array}{l}\text { Blood micro-sedi- } \\
\text { mentation, m.m. }\end{array}$} & \multirow{2}{*}{ Clinical Diagnosis } \\
\hline & & $15^{\prime}$ & $30^{\prime}$ & $45^{\prime}$ & $60^{\prime}$ & \\
\hline 1 & $6 \mathrm{y} . \quad 2 \mathrm{~m}$. & 14.0 & 17.0 & 22.0 & 25.0 & Pulmonary tuberculosis and pleuritis \\
\hline 2 & $2 \mathrm{y} .10 \mathrm{~m}$. & 2.0 & 6.5 & 10.0 & 11.0 & Influenza \\
\hline 3 & $8 \mathrm{y} .5 \mathrm{~m}$. & 1.0 & 4.5 & 7.5 & 10.0 & " \\
\hline 4 & $4 \mathrm{~m}$ & 2.0 & 4.0 & 5.5 & 7.0 & Infantile dyspepsia \\
\hline 5 & $4 \mathrm{y} . \quad 5 \mathrm{~m}$. & 9.5 & 20.0 & $22 . \overline{0}$ & 24.0 & Pleuritis \\
\hline 6 & $1 \mathrm{~g} .10 \mathrm{~m}$. & 8.5 & 12.5 & 20.5 & 23.5 & Influenza \\
\hline 7 & $6 \mathrm{y} . \quad 3 \mathrm{~m}$. & 10.0 & 16.5 & 22.0 & 24.0 & Pulmonary tuberculosis \\
\hline 8 & $6 \mathrm{y} .9 \mathrm{~m}$. & 3.5 & 13.0 & 16.0 & 18.0 & Bronchopneumonia \\
\hline 9 & $10 \mathrm{~g}$. & 2.5 & 7.5 & 12.0 & 16.5 & Soarlet fever in reconvalescence \\
\hline 10 & $8 \mathrm{y} .5 \mathrm{~m}$. & 2.0 & 5.0 & 8.5 & 10.5 & Anemia \\
\hline 11 & $2 y$. & 1.5 & 3.5 & 5.5 & 7.0 & Influenza \\
\hline 12 & $4 \mathrm{y} . \quad 4 \mathrm{~m}$ & 1.5 & 4.0 & 4.0 & 6.0 & ". \\
\hline 13 & $3 \mathrm{y} .10 \mathrm{~m}$. & 1.0 & 3.0 & 4.0 & 5.0 & Obstipation \\
\hline 14 & $9 \mathrm{y} .6 \mathrm{~m}$. & 5.5 & 11.5 & 15.0 & 17.0 & Latent tuberculosis? \\
\hline 15 & 5 $\mathrm{y} .2 \mathrm{~m}$ & 2.5 & 5.5 & 7.5 & 12.5 & Erythema nodosum? \\
\hline 16 & $4 \mathrm{y} .10 \mathrm{~m}$ & 2.0 & 3.0 & 6.5 & 7.0 & Latent tuberculosis \\
\hline 17 & $8 \mathrm{y} . \quad 2 \mathrm{~m}$. & 3.0 & 6.0 & 9.5 & 11.5 & Pleuritis (recovered) \\
\hline 18 & $10 \mathrm{y}$. & 6.0 & 7.0 & 11.0 & 14.0 & Pulmonary tuberculosis (cured) \\
\hline 19 & $2 \mathrm{y}$. & 5.0 & 13.0 & 18.0 & 22.0 & Empyema thoracis \\
\hline 20 & $2 \mathrm{y} .5 \mathrm{~m}$ & 19.0 & 24.0 & 27.0 & 27.5 & Pertussis Pneumonia Subcutaneous abscess \\
\hline 21 & $2 \mathrm{y} . \quad 1 \mathrm{~m}$ & 15.0 & 23.0 & 24.0 & 26.0 & Whooping cough pneumonia \\
\hline 22 & & 8.0 & 8.5 & 11.0 & 13.0 & Strophulus and enuresis \\
\hline 23 & $7 \mathrm{~g} .6 \mathrm{~m}$. & 1.0 & 2.0 & 6.0 & 7.5 & Hemophilia \\
\hline 24 & $9 \mathrm{~m}$ & 4.5 & 9.5 & 11.0 & 15.0 & Angina acuta \\
\hline 25 & $4 \mathrm{~m}$. & 1.0 & 2.5 & 4.0 & 7.0 & T" \\
\hline 26 & $27 \mathrm{y}$. & 1.0 & 4.0 & 7.0 & 8.5 & Infiuenza? \\
\hline 27 & $25 \mathrm{y}$. & 1.0 & 3.0 & 4.0 & 7.5 & Healthy \\
\hline 28 & $23 \mathrm{y}$. & 1.5 & 7.0 & 8.0 & 12.5 & Pharyngitis \\
\hline 29 & $4 \mathrm{y} . \quad 6 \mathrm{~m}$ & 19.0 & 24.0 & 27.0 & 27.5 & Subcutaneous abscess \\
\hline 30 & $20 \mathrm{y}$. & 5.0 & 10.0 & 18.0 & 20.5 & Pleuritis \\
\hline 31 & $5 \mathrm{y}$. & 1.5 & 4.0 & 8.0 & 10.0 & Bronchitis \\
\hline 32 & $6 \mathrm{y}$. & 5.0 & 13.0 & 19.0 & 24.0 & Intestinal tuberculosis \\
\hline 33 & $10 \mathrm{~g}$. & 3.5 & 7.0 & 13.0 & 17.0 & Pulmonary tuberculosis \\
\hline 34 & $9 \mathrm{y}$. & 15.0 & 20.5 & 23.5 & 25.6 & Peritonitis \\
\hline 35 & $8 \mathrm{y} .5 \mathrm{~m}$. & 8.0 & 13.0 & 18.5 & 21.0 & Pleuroperitonitis \\
\hline 36 & $5 \mathrm{y} .10 \mathrm{~m}$. & 14.0 & 19.0 & 22.5 & 25.5 & Bronchopneumonia, Pleuritis \\
\hline 37 & $6 \mathrm{y} .4 \mathrm{~m}$. & 12.0 & 23.0 & 27.0 & 30.0 & Pleuritis \\
\hline 38 & $9 \mathrm{~g} .2 \mathrm{~m}$. & 3.5 & 9.5 & 14.0 & 19.0 & Peritonitis \\
\hline 39 & $6 \mathrm{y}$. & 8.0 & 12.0 & 18.0 & 21.5 & Pleuritis \\
\hline 40 & $7 \mathrm{y} .1 \mathrm{~m}$. & 6.0 & 22.0 & 26.0 & 28.0 & Peritonitis, Pulmonary tuberculosis \\
\hline 41 & 9 у. & 1.0 & 5.0 & 10.0 & 15.0 & Nephritis \\
\hline 42 & $5 \mathrm{y} . \quad 2 \mathrm{~m}$. & 6.0 & 11.5 & 15.5 & 23.0 & Otitis media acuta \\
\hline 43 & $23 y$ & 0.5 & 1.5 & 2.5 & 4.5 & Healthy \\
\hline 44 & $14 \mathrm{y}$. & 1.3 & 4.0 & 6.5 & 8.5 & Influenza. \\
\hline 45 & $1 \mathrm{y}$. & 1.5 & 2.5 & 4.5 & 7.0 & Dystrophia \\
\hline 46 & $16 \mathrm{y}$. & 1.0 & 1.5 & 2.5 & 4.0 & Healthy \\
\hline 47 & $24 \mathrm{~g}$. & 1.5 & 4.5 & 9.5 & 11.0 & Keratitis parechymatosa \\
\hline 48 & $9 \mathrm{y}$. & 12.0 & 23.0 & 26.0 & 28.0 & Pulmonary tuberculosis \\
\hline 49 & 4 g. $8 \mathrm{~m}$. & 4.0 & 16.0 & 22.0 & 26.0 & Pleuritis \\
\hline 50 & $7 \mathrm{y} .8 \mathrm{~m}$. & 2.0 & 9.0 & 16.0 & 18.0 & Pulmonary tuberculosis \\
\hline $\mathbf{5 1}$ & $4 \mathrm{y} .4 \mathrm{~m}$ & 1.5 & 4.0 & 7.0 & 10.0 & Latent tuberculosis \\
\hline
\end{tabular}




\begin{tabular}{|c|c|c|c|c|c|c|}
\hline \multirow{2}{*}{$\begin{array}{c}\text { Nos. } \\
\text { of } \\
\text { cases }\end{array}$} & \multirow{2}{*}{ Age } & \multicolumn{4}{|c|}{$\begin{array}{l}\text { Blood micro-sedi- } \\
\text { mentation, m. m. }\end{array}$} & \multirow{2}{*}{ Clinical Diagnosis } \\
\hline & & $15^{\prime}$ & $30^{\prime}$ & $45^{\prime}$ & $60^{\prime}$ & \\
\hline 52 & $3 \mathrm{~g} .9 \mathrm{~m}$. & 8.0 & 17.0 & 20.0 & 21.0 & Bronchopneumonia \\
\hline 53 & $1 \mathrm{~g} .5 \mathrm{~m}$. & 2.0 & 6.5 & 13.0 & 17.5 & Pneumonia \\
\hline 54 & $10 y$. & 9.0 & 14.0 & 18.0 & 21.0 & Pleuroperitonitis \\
\hline 55 & $9 y$. & 1.0 & 3.0 & 6.0 & 8.0 & Influenza \\
\hline 56 & $8 \mathrm{~g} .4 \mathrm{~m}$. & 3.3 & 15.5 & 23.0 & 25.5 & Scarlet fever \\
\hline
\end{tabular}

\section{TABLE IV.}

Blood micro-sedimentation in different cases.

\begin{tabular}{|c|c|c|c|c|}
\hline \multirow{2}{*}{$\begin{array}{c}\text { Nos. } \\
\text { of } \\
\text { cases }\end{array}$} & \multirow{2}{*}{ Age } & \multicolumn{2}{|c|}{$\begin{array}{c}\text { Sedimentation, } \\
\text { m.m. }\end{array}$} & \multirow{2}{*}{ Clinical diagnosis } \\
\hline & & $30^{\prime}$ & $60^{\prime}$ & \\
\hline 1 & 6 y. $9 \mathrm{~m}$. & 6.0 & 12.0 & Hilus tuberculosis \\
\hline 2 & $11 \mathrm{~g}$. & 5.7 & 11.0 & Traumatic pleuritis (recovered) \\
\hline $\mathbf{3}$ & $7 \mathrm{~g} . \quad 3 \mathrm{~m}$ & 11.0 & 17.5 & Pleuritis \\
\hline 4 & 2 g. $10 \mathrm{~m}$. & 5.0 & 10.9 & Latent tuberculosis \\
\hline $\mathbf{5}$ & $2 \mathrm{~g}, \quad 1 \mathrm{~m}$ & 5.5 & 14.0 & Conjunctivitis phlyctenulosa \\
\hline 6 & $7 \mathrm{y} . \quad 8 \mathrm{~m}$ & 3.5 & 8.0 & Healthy \\
\hline 7 & $5 \mathrm{~s} .2 \mathrm{~m}$. & 3.0 & 7.0 & $"$ \\
\hline 8 & $13 \mathrm{~g}$ & 6.0 & 11.5 & Apicitis tuberculosa? \\
\hline 9 & $11 \mathrm{y} .5 \mathrm{~m}$ & 5.0 & 10.0 & Latent tuberclosis \\
\hline 10 & $8 \mathrm{y} .2 \mathrm{~m}$ & 15.5 & 22.5 & Phthisis pulmonum tuberculosa \\
\hline 11 & $12 \mathrm{y}$. & 23.0 & 26.5 & Pulmonary tuberculosis \\
\hline 12 & $9 \mathrm{y}$. & 7.0 & 18.5 & Pleuritis \\
\hline 13 & $9 \mathrm{y} . \quad 6 \mathrm{~m}$ & 4.0 & 11.5 & Bronchoadenitis? \\
\hline 14 & $5 \mathrm{~g} .9 \mathrm{~m}$ & 2.5 & 5.5 & Healthy \\
\hline 15 & $8 \mathrm{~g}$. & 2.5 & 5.0 & Spondylitis taberculosa? \\
\hline 16 & $8 \mathrm{y} .5 \mathrm{~m}$ & 17.5 & 23.0 & Pleuritis \\
\hline 17 & $12 \mathrm{y} .3 \mathrm{~m}$. & 1.5 & 4.0 & Anorexia nervosum \\
\hline 18 & $10 \mathrm{y} .8 \mathrm{~m}$ & 21.5 & 25.5 & Pulmonary tuberculosis \\
\hline 19 & $8 \mathrm{y.}$ & 2.0 & 8.0 & Gastroptosis \\
\hline 20 & $12 \mathrm{y} .8 \mathrm{~m}$. & 16.5 & 25.0 & Pleuroperitonitis \\
\hline 21 & 3 у. $4 \mathrm{~m}$. & 6.0 & 20.0 & Chronic blonchopneumonia \\
\hline 22 & $6 \mathrm{y} .6 \mathrm{~m}$ & 10.0 & 16.5 & Chronic bronchitis \\
\hline 23 & $7 y$. & $\mathbf{3 . 5}$ & 7.5 & Rhinitis \\
\hline 24 & $3 \mathrm{y} .7 \mathrm{~m}$ & 2.0 & 6.5 & Cystitis? \\
\hline 25 & $2 \mathrm{y} .11 \mathrm{~m}$ & 5.5 & 10.5 & Chronic dyspepsia \\
\hline 26 & $7 \mathrm{y} .11 \mathrm{~m}$ & 11.5 & 20.0 & Endocarditis \\
\hline 27 & $5 \mathrm{y}$ & 12.0 & 24.5 & Pleuroperitonitis tuberculosa \\
\hline 28 & $4 \mathrm{y.} \quad 9 \mathrm{~m}$ & 11.0 & 17.5 & Pulmonary tuberculosis \\
\hline 29 & $3 \mathrm{y} . \quad 6 \mathrm{~m}$ & 6.0 & 11.5 & Latent tuberculosis \\
\hline 30 & $5 \mathrm{y} . \quad 2 \mathrm{~m}$ & 7.0 & 17.0 & Lymphadenitis colli \\
\hline 31 & $9 \mathrm{y} . \quad 4 \mathrm{~m}$ & 18.0 & 26.0 & Pleuroperitonitis \\
\hline 32 & $3 \mathrm{y.} \quad 8 \mathrm{~m}$ & 7.0 & 15.5 & Enuresis, Hilitis tuberculosa \\
\hline 33 & $11 \mathrm{y}$. & 3.0 & 8.0 & Hyperventilation \\
\hline 34 & 5 y. $4 \mathrm{~m}$. & 3.5 & 8.5 & Latent tuberculosis \\
\hline 35 & $8 \mathrm{y}$. & 8.5 & 15.0 & Brohchitis acuta \\
\hline 36 & 2 у. $6 \mathrm{~m}$. & 21.0 & 24.5 & Pulmonary tuberculosis \\
\hline 37 & $2 \mathrm{y} . \quad 5 \mathrm{~m}$ & 9.0 & 16.5 & Chronic bronchitis \\
\hline 38 & $7 \mathrm{y} .10 \mathrm{~m}$ & 5.5 & 12.0 & Hilitis tuberculosa \\
\hline
\end{tabular}




\begin{tabular}{|c|c|c|c|c|}
\hline \multirow{2}{*}{$\begin{array}{c}\text { Nos. } \\
\text { of } \\
\text { cases }\end{array}$} & \multirow[t]{2}{*}{ Age } & \multicolumn{2}{|c|}{$\begin{array}{l}\text { Sedimentation, } \\
\text { m.m. }\end{array}$} & \multirow[t]{2}{*}{ Clinical diagnosis } \\
\hline & & $30^{\prime}$ & $60^{\prime}$ & \\
\hline 39 & $6 \mathrm{y} .1 \mathrm{~m}$ & 3.5 & 10.0 & Enuresis nocturna \\
\hline 40 & $10 \mathrm{y} .5 \mathrm{~m}$. & 14.5 & 21.0 & Typhus abdominalis \\
\hline 41 & 7 ร. $10 \mathrm{~m}$ & 9.0 & 16.0 & Chronic bronchitis \\
\hline 42 & $5 \mathrm{y} .8 \mathrm{~m}$ & 3.0 & 15.5 & Anorexia \\
\hline 43 & $12 \mathrm{~J}$. & 26.5 & 29.0 & Pulmonary tuberculosis \\
\hline 44 & $5 \mathrm{y}$. & 11.5 & 20.0 & Chronic bronchitis \\
\hline 45 & $8 \mathrm{y} .5 \mathrm{~m}$. & $\mathbf{7 . 0}$ & 15.5 & Pulmonary tuberculosis \\
\hline 46 & $8 \mathrm{~g} . \quad 3 \mathrm{~m}$. & 6.5 & 13.5 & Syphilis \\
\hline 47 & $6 \mathrm{~g} .10 \mathrm{~m}$ & 7.0 & 12.5 & Peritonitis \\
\hline 48 & $3 \mathrm{y} . \quad 8 \mathrm{~m}$ & 6.0 & 11.0 & Cardiac disease \\
\hline 49 & $9 \mathrm{y.} 8 \mathrm{~m}$. & 13.0 & 21.0 & Pleuropneamonia tuberculosa \\
\hline 50 & $3 \mathrm{~g} . \quad 1 \mathrm{~m}$. & 18.0 & 23.0 & Tuberculeuse intestinal stenosis \\
\hline 51 & $5 \mathrm{~g} .1 \mathrm{~m}$ & 3.0 & 6.5 & Traumatic neuralgia \\
\hline 52 & $5 \mathrm{~g} .9 \mathrm{~m}$. & 5.0 & 9.5 & Bronchoadenitis tuberculosa \\
\hline 53 & 5 у. $7 \mathrm{~m}$. & 5.0 & 11.5 & Pulmonary affection. \\
\hline 54 & $10 \mathrm{y} .10 \mathrm{~m}$ & 18.5 & 25.0 & Pulmo-intestinal tuberculosis \\
\hline 55 & 8 y. $6 \mathrm{~m}$ & 145 & 23.0 & Brain tumor, Bronchitis (glioma) \\
\hline 56 & $8 \mathrm{y} .1 \mathrm{~m}$. & 15.0 & 21.0 & Pulmonary tuberculosis \\
\hline 57 & $8 \mathrm{y}$. & 6.0 & 12.0 & Otitis media chronica \\
\hline 58 & $1 \mathrm{~g} . \quad 2 \mathrm{~m}$. & 4.0 & 9.5 & Anemia \\
\hline 59 & 4 у. $3 \mathrm{~m}$ & 6.0 & 15.5 & Asthmatic bronchitis \\
\hline 60 & $3 \mathrm{g.} \quad 9 \mathrm{~m}$. & 13.0 & 22.0 & Pleuritis \\
\hline 61 & 5 g. $9 \mathrm{~m}$. & 5.6 & 14.0 & Lymphadenitis colli \\
\hline 62 & 4 y. $7 \mathrm{~m}$. & 6.0 & 13.0 & Intestinal tuberculosis \\
\hline 63 & $3 y$. & 1.5 & 4.0 & Genuine epilepsia \\
\hline 64 & $4 \mathrm{y} .11 \mathrm{~m}$ & 2.5 & 4.5 & Nephritis impetiginosa \\
\hline 65 & $10 \mathrm{y}$. & 4.0 & 7.0 & Healthy \\
\hline 66 & $3 \mathrm{y}$. & 3.0 & 12.5 & Diphtherie postparesis \\
\hline 67 & $1 \mathrm{~g} .8 \mathrm{~m}$ & 16.0 & 21.0 & Tracheobronchitis \\
\hline 68 & 9 у. $8 \mathrm{~m}$. & 11.0 & 18.5 & Parotitis epidemica \\
\hline 69 & 5 y. $8 \mathrm{~m}$. & 2.5 & 10.0 & Influenza \\
\hline 70 & $5 \mathrm{~g} .4 \mathrm{~m}$. & 8.5 & 18.5 & Enteritis acuta. Cystitis \\
\hline 71 & $6 \mathrm{y} .3 \mathrm{~m}$. & 1.5 & 4.5 & Latent tubercnlosis? \\
\hline 72 & $8 \mathrm{y} .10 \mathrm{~m}$. & 10.5 & 18.5 & Pulmonary tuberculosis \\
\hline 73 & $6 \mathrm{y} .7 \mathrm{~m}$. & 19.5 & 30.0 & Scarlet fever \\
\hline 74 & $8 \mathrm{y} .4 \mathrm{~m}$. & 14.0 & 22.5 & Peritonitis, Lymphadenitis tuberculosa \\
\hline 75 & $7 \mathrm{~m}$ & 2.5 & 6.0 & Avitaminotic dyspepsia \\
\hline 76 & $10 \mathrm{y.} 8 \mathrm{~m}$ & 6.0 & 11.5 & Ascariasis \\
\hline 77 & $10 \mathrm{y} .5 \mathrm{~m}$ & 14.0 & 17.0 & Dysentheria \\
\hline 78 & $6 \mathrm{s.} 10 \mathrm{~m}$ & 15.0 & 20.5 & Scarlet fever \\
\hline 79 & 14 y. $9 \mathrm{~m}$. & 5.0 & 9.0 & Influenza \\
\hline 80 & $8 \mathrm{y} .6 \mathrm{~m}$. & 9.5 & 17.0 & Thyphoid fever \\
\hline 81 & $2 \mathrm{y} .4 \mathrm{~m}$. & 5.0 & 15.0 & Iridocyclitis \\
\hline 82 & $11 \mathrm{~m}$. & 25.0 & 28.5 & Infantile dyspepsia with atrophia \\
\hline 83 & $8 \mathrm{y} .10 \mathrm{~m}$ & 2.0 & 9.0 & Influenza \\
\hline 84 & $5 \mathrm{g.} \quad 5 \mathrm{~m}$. & 1.5 & 2.5 & $\begin{array}{l}\text { Pleuroperitonitis with Phthisis pulmonum } \\
\text { tuberculosa (before } 3 \text { days of her death) }\end{array}$ \\
\hline 85 & 11 у. $7 \mathrm{~m}$. & 3.0 & 8.5 & Spondylitis cariosa \\
\hline 86 & $6 \mathrm{y}$. & 6.0 & 10.5 & Morbus Addissonii \\
\hline 87 & $8 \mathrm{y} . \quad 2 \mathrm{~m}$. & 16.0 & 20.5 & Pollakisuria \\
\hline 88 & $7 \mathrm{~g} .9 \mathrm{~m}$. & 21.0 & 27.0 & Pulmonary tuberculosis with Pleuritis \\
\hline 89 & $6 \mathrm{y.} 2 \mathrm{~m}$. & 23.0 & 26.0 & Pleuritis exsudativa \\
\hline 90 & $10 \mathrm{~m}$ & 3.0 & 7.0 & Influenza \\
\hline 91 & $5 \mathrm{~g}$. & 6.5 & 13.5 & Chicken pox \\
\hline 92 & $5 \mathrm{~g} .7 \mathrm{~m}$. & 26.5 & 28.0 & Otitis media suppurativa acuta \\
\hline
\end{tabular}




\begin{tabular}{|c|c|c|c|c|}
\hline \multirow{2}{*}{$\begin{array}{c}\text { Nos. } \\
\text { of } \\
\text { cases }\end{array}$} & \multirow{2}{*}{ Age } & \multicolumn{2}{|c|}{$\begin{array}{c}\text { Sedimentation, } \\
\mathrm{m} . \mathrm{m} \text {. }\end{array}$} & \multirow[t]{2}{*}{ Clinical diagnosis } \\
\hline & & $30^{\prime}$ & $60^{\prime}$ & \\
\hline 93 & $4 \mathrm{y} . \quad 5 \mathrm{~m}$ & 4.5 & 10.0 & Latent tuberculosis \\
\hline 94 & $5 \mathrm{y}, 3 \mathrm{~m}$. & 4.5 & 8.5 & Healthy \\
\hline 95 & $5 \mathrm{y} .8 \mathrm{~m}$. & 6.5 & 16.5 & Rheumatism \\
\hline 96 & $3 \mathrm{~g} . \quad 2 \mathrm{~m}$ & 9.0 & 19.0 & Bronchopneumenia \\
\hline 97 & $1 \mathrm{~g} .5 \mathrm{~m}$ & 23.0 & 28.0 &, \\
\hline 98 & $1 \mathrm{y} . \quad 1 \mathrm{~m}$. & 2.0 & 7.5 & Poliomyelitis anterior \\
\hline 99 & $3 \mathrm{~m}$ & 6.0 & 19.0 & Bronchopneumonia \\
\hline 100 & $1.5 \mathrm{~m}$. & 18.0 & 24.0 & Hereditary syphilis \\
\hline 101 & 4 у. $9 \mathrm{~m}$. & 4.0 & 8.0 & Influenza \\
\hline 102 & 9 у. $2 \mathrm{~m}$. & 25.0 & 26.5 & Pulmonary tuberculosis \\
\hline 103 & $10 \mathrm{~g} .2 \mathrm{~m}$. & 5.0 & 11.0 & Taeniasis \\
\hline 104 & $8 \mathrm{~m}$ & 19.0 & 23.0 & Bronchopneumonia \\
\hline 105 & $7 \mathrm{y} .10 \mathrm{~m}$. & 5.0 & 16.5 & Mastoiditis acuta Intestinal tuberculosis? \\
\hline 106 & $8 \mathrm{~g} .9 \mathrm{~m}$. & 2.0 & 9.0 & Intestinal tuberculosis? \\
\hline 107 & $5 \mathrm{y}$. & 11.5 & 15.5 & Bronchoadenitis tuberculosa \\
\hline 108 & $18 \mathrm{y}$. & 9.0 & 11.0 & Paroxymale hemoglobinuria \\
\hline 109 & $8 \mathrm{y} . \quad 2 \mathrm{~m}$. & 2.0 & 5.0 & Angina diphtherica \\
\hline
\end{tabular}

\section{Conclusions.}

1) A new micro method for hemosedimentation has been described. The method needs only 0.05 c.c. of blood for a sample. The rack is portable anywhere with blood-filled pipettes.

2) This method has an average deviation of $\pm 0.7 \mathrm{~mm}$. from Langer-Schmidt's micro method.

3) This method is compared with Westergren's micro method and there is a fair parallelism between the two methods. 